\title{
Depression of insulator charging in vacuum by partial mechanical processing
}

\author{
$\operatorname{AUTHOR}(\mathrm{S})$ :
}

Yamamoto, O; Markon, S; Morii, H

\section{CITATION:}

Yamamoto, O ...[et al]. Depression of insulator charging in vacuum by partial mechanical processing. IEEE TRANSACTIONS ON DIELECTRICS AND ELECTRICAL INSULATION 2007, 14(3): 606-612

\section{ISSUE DATE:}

2007-06

URL:

http://hdl.handle.net/2433/50114

\section{RIGHT:}

(c)2007 IEEE. Personal use of this material is permitted. However, permission to reprint/republish this material for advertising or promotional purposes or for creating new collective works for resale or redistribution to servers or lists, or to reuse any copyrighted component of this work in other works must be obtained from the IEEE. 


\title{
Depression of Insulator Charging in Vacuum by Partial Mechanical Processing
}

\author{
Osamu Yamamoto \\ Kyoto University \\ Kyoto, Kyoto 615-8510, Japan \\ Sandor Markon \\ Kobe Institute of Technology, Graduate School of Information Technology \\ Kano-cho, Chuo-ku, Kobe 650-0001, Japan \\ and Hiroshi Morii \\ Power Engineering R \& D Center, Kansai Electric Power Co Inc. \\ Amagasaki, Hyogo 661-0974, Japan
}

\begin{abstract}
It has been clarified by researchers that insulators having contact angles, e.g. $\pm 45^{\circ}$ with regard to the plane electrodes, is hard to acquire charge when they are exposed to high voltages in vacuum. For example, an insulator in the shape of a truncated frustum is free from charging. However, since such insulator has inevitably large volume, it obstructs the compactness. On the other hand, roughening insulator surface is also effective to prevent the surface from charging. In this case, the cost for roughening the entire surface may be high when the insulator is large. In this paper we review our previous investigation results and describe new experimental results aimed at developing feasible and compact insulators used in vacuum. Surface charging characteristics of partially and mechanically processed insulators have been studied for this purpose. That is, the charging phenomena when such insulators are exposed to a ramped dc or ac voltage have been observed by using a capacitive probe embedded in the cathode. Firstly, we describe of a partially roughened cylindrical insulator, where the height of the roughened surface measured from the cathode is varied keeping the total length constant. Secondly, we describe a cylindrical insulator having a truncated conical frustum or a chamfer at its cathode-side end. Based on these experimental and simulation results we prove that the partial mechanical processing is effective to control and suppress the insulator charging in vacuum.
\end{abstract}

Index Terms = Surface charge, flashover, depression and control of charging, $\mathbf{d c}$ and ac high voltages, roughening, truncated conical frustum, partial processing, vacuum.

\section{INTRODUCTION}

BESIDES vacuum circuit breakers, equipment requiring insulation of high voltages or high electric fields in vacuum include generators and accelerators of charged particle beams like electrons, ions, heavy ions; pulsed power equipment; electron microscopes; microwave tubes; X-ray tubes; and others. Furthermore, high-voltage insulation is also needed in the development of new generation flat panel displays (FPD). Another related problem is the insulation of the cover glass of solar cell arrays for satellites [1,2]. In such vacuum equipment, the insulation problem involves not only the vacuum gap between the metallic electrodes, but also the supporting solid insulators (called 'spacers' below) in parallel with that. Generally, the insulation strength of surfaces is

Manuscript received on 25 July 2006, in final form 11 December 2007. extremely poor, and the discharge voltage (or flashover voltage) shows considerable saturation with respect to the insulation length; thus great care is needed in the design of vacuum insulations.

The materials used for the spacers holding the high-voltage electrodes in these equipment is usually alumina or other ceramics, but polymers or glasses are also used, depending on the environmental requirements, cost, forming and machining properties etc. Their shape can be cylindrical or angular for internal parts, or tubular when used as the vacuum vessel.

In the vacuum region, the electrons emitted due to extremely high field caused at the imperfect contact areas between the cathode and the insulators (triple points) impact on the insulator, and cause surface charging of the insulator through the SEEA (Secondary Electron Emission 


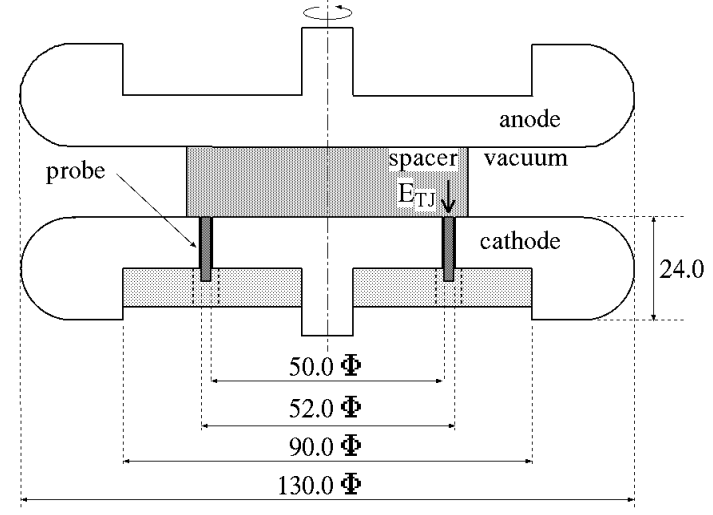

[mm]

Figure 1. Arrangement of insulator specimen and probe

Avalanche) process [3] and thus surface discharge. Therefore, much effort have been put into the study of methods for controlling the secondary electron emission characteristics of the spacer surfaces [4] as well as into finding such shapes for the spacer that make them difficult to impact on the surface (i.e. into improving the electric field distribution) [5,6], which are the main approaches used to reduce the charging effect. We report here our theoretical and experimental results concerning the reduction of charging.

\section{Charging Process Observed by Probe}

We have adopted the probe arrangement shown in Figure1 to observe the charging process in real time [7]. In order to avoid influencing the electric field in the discharging region, part of the surface of the cathode is insulated. Furthermore, to prevent electrons or ions directly entering the probe, it is embedded in the insulator test piece. The shape of the probe is adjusted to the exterior shape of the insulator, and made into a ring. In case of a test piece with relatively small diameter, the charging effect is

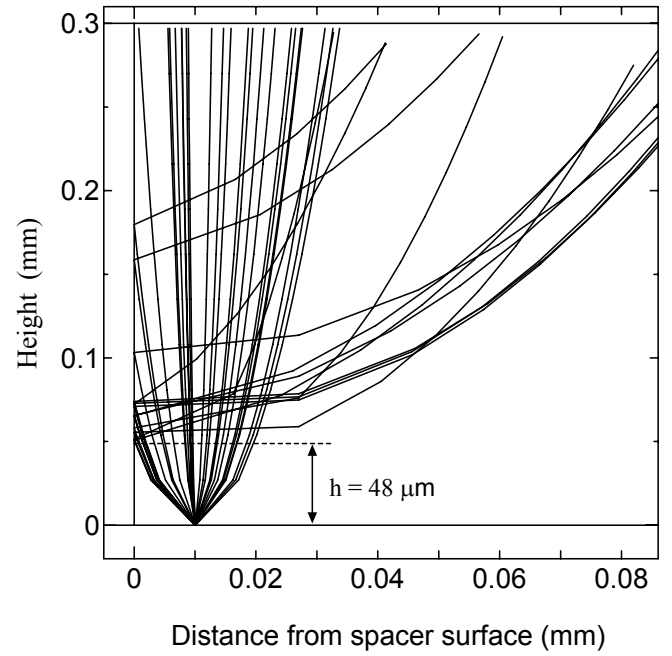

(a)

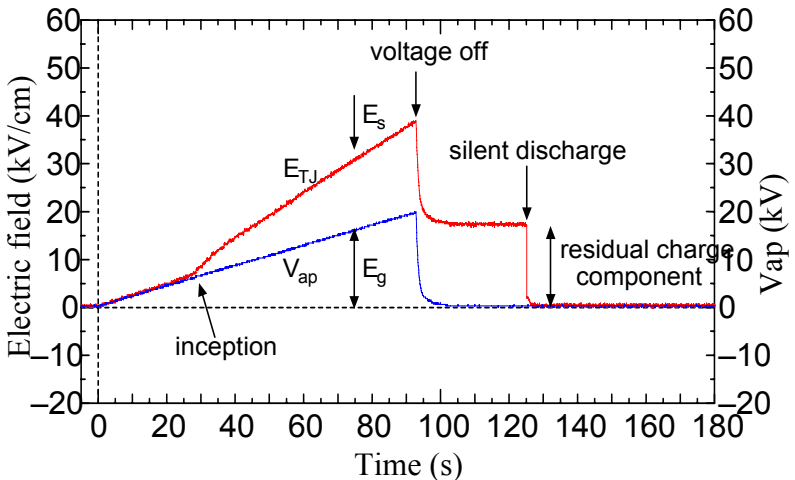

Figure 2. An example of charge measurement when a cylindrical insulator is subjected to a ramped voltage[8]. (PMMA. with smooth surface. $54 \mathrm{~mm} \phi, 10 \mathrm{~mm}$ length).

usually even in the peripheral direction [8] (that is, it is circularly symmetrical), thus the ring shape is appropriate. It is possible to treat the case of peripherally uneven distributions, by subdividing the ring into segments [9]. In any case, the probe is connected to a condenser, and the measurement signal is obtained from its terminal voltage. By converting this terminal voltage to the electric field, it will be the sum of the applied field $E_{g}$, and the field $E_{s}$ due to the surface charge [7].

Since we locate the probe near to the cathode triple junction, as close as possible, we denote the summation of the fields as $\mathrm{E}_{\mathrm{TJ}} ; \mathrm{E}_{\mathrm{s}}+\mathrm{E}_{\mathrm{g}} \equiv \mathrm{E}_{\mathrm{TJ}}$. $\mathrm{E}_{\mathrm{s}}, \mathrm{E}_{\mathrm{g}}$ and thus $\mathrm{E}_{\mathrm{TJ}}$ are average electric fields on the probe located close to the triple junction and is not identical to the field at the triple point. The electric field strength at triple point due to the surface charge can be almost ten times higher than the measured $E_{\mathrm{s}}$ when we calculate the field strength taking a simulated surface charge distribution in to account [10].

In Figure 2, we show an example of the charging process observed by using the probe. In this graph, we show the probe signal $\mathrm{E}_{\mathrm{TJ}}$ measuring the electric field in the vicinity of the triple point at the cathode surface, for the case of a

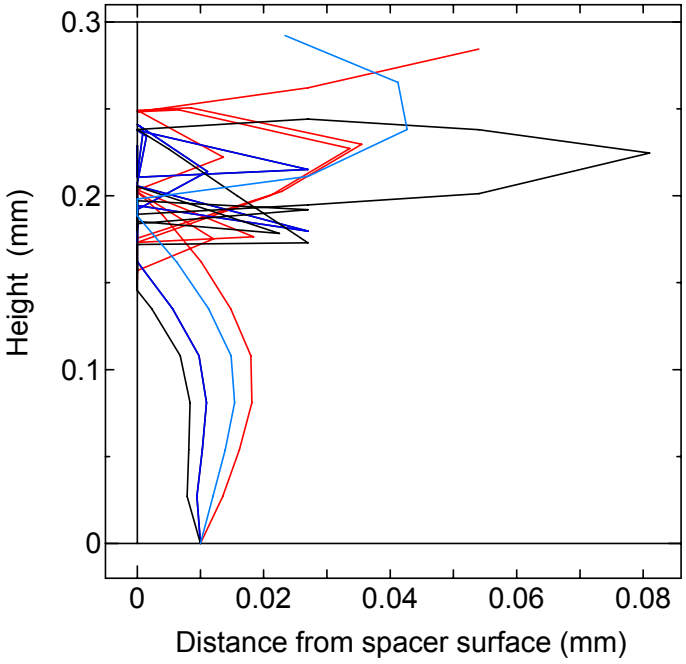

(b)

Figure 3. Calculated electron trajectories at (a) an early stage, and (b) a middle stage of charging process of a cylindrical insulator. (PMMA. 54 mm diameter, 10 mm length.) 


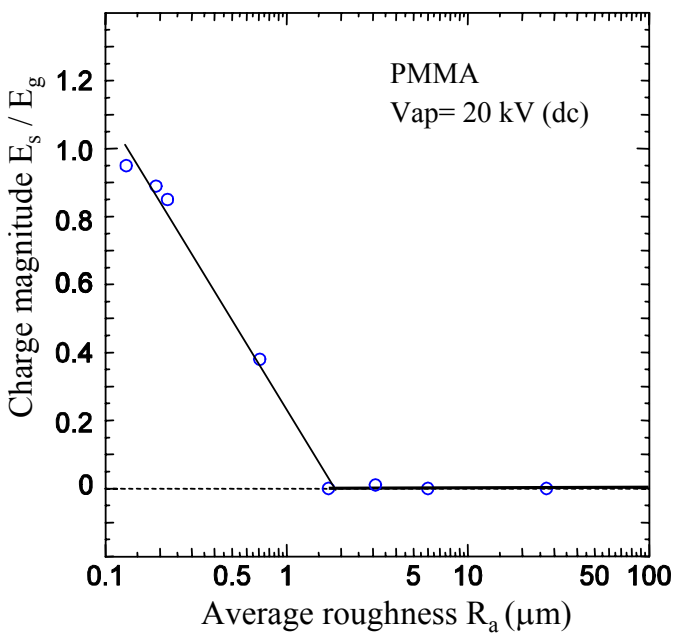

Figure 4. Relation of normalized charge magnitude with surface roughness for a cylindrical insulator.

ramped dc voltage acting on a cylindrical test piece (diameter $54 \mathrm{~mm}$, length $10 \mathrm{~mm}$, average surface roughness $\mathrm{R}_{\mathrm{a}}=0.17 \mu \mathrm{m}$ ). Thus $\mathrm{E}_{\mathrm{TJ}}$ is the sum of the external field component $\mathrm{E}_{\mathrm{g}}$ and the charging field component $\mathrm{E}_{\mathrm{s}}$. As the voltage is increased, at reaching a certain point, charging will begin, and $E_{s}$ is superposed on $E_{g}$. It is apparent that $E_{s}$ is approximately proportional to the terminal voltage, which agrees with the charging theory according to the SEE mechanism [3]. When we remove the terminal voltage (in Figure 2, it is $20 \mathrm{kV}$ ), $\mathrm{E}_{\mathrm{g}}$ becomes zero, but the $\mathrm{E}_{\mathrm{s}}$ component remains, indicating that the test piece stays charged. Subsequently, when a small amount of e.g. air is let into the vacuum chamber, the electric field of the remaining charges causes low-pressure discharge (denoted as 'Silent discharge' in the graph), and the charge is readily dispersed. From this phenomenon, we can assume that only the surface had been charged.

\section{EFFECT OF SURFACE ROUGHNESS}

\subsection{SIMULATION}

One very simple and effective method of controlling the charging effect is by increasing the surface roughness of the spacer [11]. If we simulate the path of the electrons emitted from the cathode, an enlarged image will look like Figure 3. Figure 3a shows the state at the beginning of the charging, when some of the electrons impinge on the spacer surface, and the secondary electrons move toward the anode without re-entering the spacer. As the surface charging progresses, the field due to the positive surface charges prevents the secondary electrons from leaving, with the height of the resulting path (the so-called 'Hopping height') staying in the range of a few tens of $\mu$ meters from the surface. Figure $3 b$ shows this situation, where the trajectories of 'hopping' secondary, tertiary, $\cdots$ electrons, that eventually become an avalanche. When the charging progresses still further, the

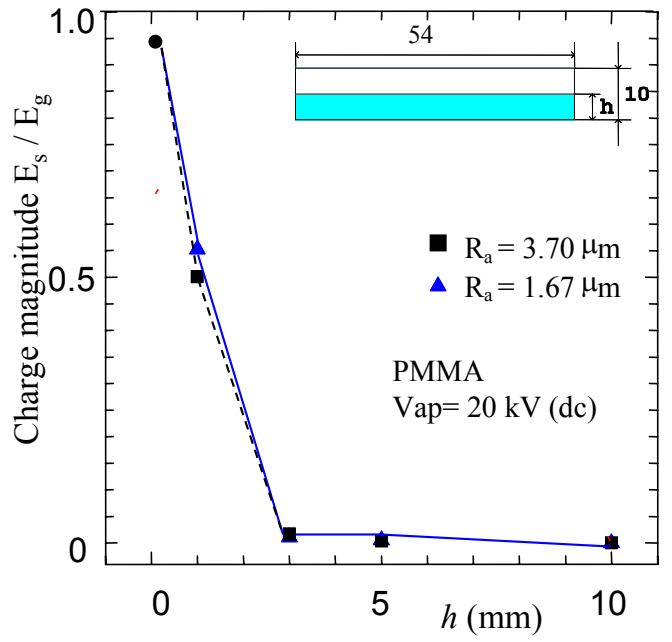

Figure 5. Relation of normalized charge magnitude with height (h) of partially roughened surface.

height of the path from the surface gets at or below the 1 $\mu \mathrm{m}$ range [11]. Therefore, if the surface has larger irregularities than this, it is expected that the electrons will not be able to pass over them.

\subsection{EXPERIMENTS CONCERNING THE EFFECT OF SURFACE ROUGHNESS}

In Figure 4, we show experimental results obtained with varying the surface roughness of test pieces from about 0.1 $\mu \mathrm{m}$ to a few tens of $\mu \mathrm{m}$. The abscissa is $E_{\mathrm{s}}$, normalized with $\mathrm{E}_{\mathrm{g}}$, indicating the charging strength. As this example shows, the charging strength decreases with increasing surface roughness, and in case of PMMA, above around 2 $\mu \mathrm{m}$, we get $\mathrm{E}_{\mathrm{s}} / \mathrm{E}_{\mathrm{g}}=0$, that is, charging no longer occurs. When we consider further the experimental results obtained with glass and alumina, we can say that above around 3 $\mu \mathrm{m}$, we can practically control the charging process, irrespective of the material [11]. At an average surface roughness of around $3 \mu \mathrm{m}$, the insulating strength will improve by about a factor of 2 .

\subsection{THE EFFECT OF PARTIAL ROUGHENING}

In order to find out what part of the spacer needs to be roughened to be effective, we have studied the charging characteristics of spacers with their surface roughened at distances of $h$ from the cathode. The result is shown in Figure 5. In this experiment, the roughness of the area within the height $h$ was set to either to $R_{a}=1.67$ or to 3.70 $\mu \mathrm{m}$, with the rest set to $\mathrm{R}_{\mathrm{a}}=0.17 \mu \mathrm{m}$. As it is seen from the graph, it is sufficient to roughen an area within $h=3 \mathrm{~mm}$ in order to achieve a charging control effect.

In the case when a part of the anode side surface was roughened, the $E_{s} / E_{g}$ increased with the decrease in the roughened area. 


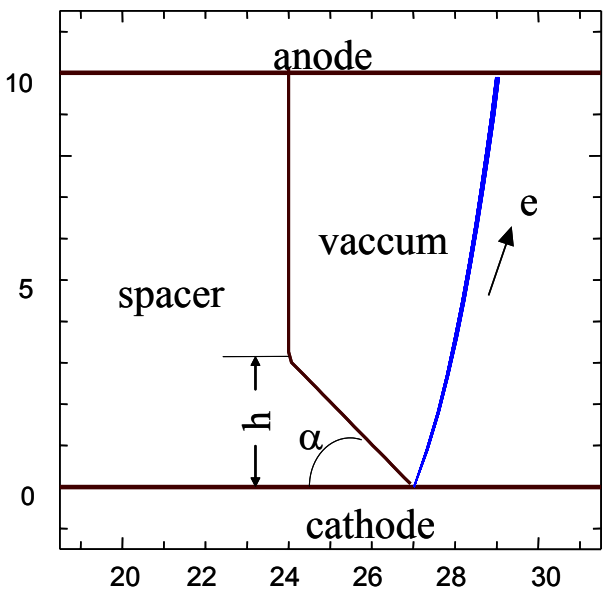

Figure 6. An insulating spacer model with a frustum at the cathode end. (PMMA. $\alpha=45^{\circ}, \mathrm{h}=3 \mathrm{~mm}$ )).

\section{EFFECT OF THE SPACER SHAPE}

When the spacer surface is perpendicular to the cathode, the electric field strength in the vicinity of the triple point is proportional to the applied voltage $\mathrm{V}_{\mathrm{ap}}$, divided by the gap distance $\mathrm{d}$ equaling to the spacer height. That is, $\mathrm{E}_{\mathrm{g}}=\mathrm{V}_{\mathrm{ap}} / \mathrm{d}$. However, if the cathode and the surface of the spacer are conically shaped and meet in an obtuse angle, the electric field strength at the triple point can be reduced considerably from $V_{\text {ap }} / d$. Furthermore, in such case the electric field in the vacuum changes its direction so that it will repel the electrons from the spacer surface, thus they will not impinge on the surface and thus will not contribute to the charging. Therefore, the charging component will become zero, and if we perform the same measurement like in Figure 2, $\mathrm{E}_{\mathrm{g}}$ and $\mathrm{E}_{\mathrm{TJ}}$ will overlap [12].

However, as it can be easily seen, if the supporting spacer is made conical, the volume occupied by it will be large, conflicting with the goal of making the vessel compact. Therefore, we need to find a way to prevent the growth of the spacer size.

We have therefore investigated the charging reduction effect for the case when instead of making the whole spacer

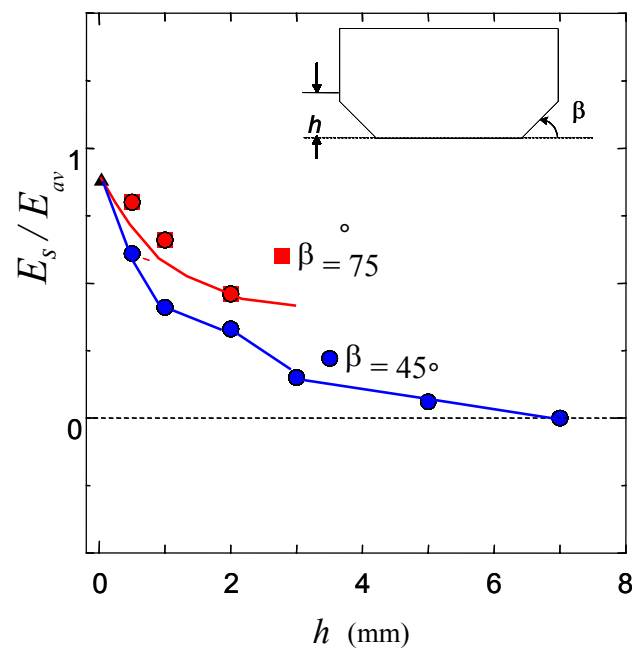

Figure 8. Relation of charge magnitude with $\mathrm{h}$. (PMMA, $\left.\mathrm{R}_{\mathrm{a}}=0.17 \mu \mathrm{m}\right)$.

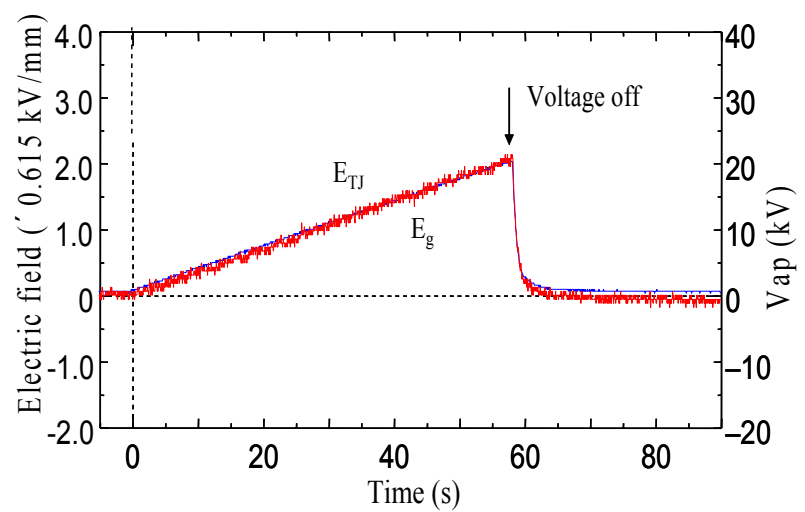

Figure 7. An example of charge measurement when a cylindrical insulator with a frustum is subjected to a ramped voltage. (PMMA. $\alpha=45^{\circ}, \mathrm{h}=3$ $\left.\mathrm{mm}, \mathrm{R}_{\mathrm{a}}=0.17 \mu \mathrm{m}\right)$.

conical, it remains cylindrical, except for the region connecting with cathode where it is made conical. The compactness of the spacer depends on how tall this conical part is. In our case, the total height of the spacer is $10 \mathrm{~mm}$, and we have varied the height $h$ of the conical part from 3 to $10 \mathrm{~mm}$. In Figure 6, we show the simulated electron path for the case of $h=3 \mathrm{~mm}$, from where it becomes clear that even if only part of the spacer is made conical, it still has an inhibitive effect on the impacting of electrons. Also, from our measurements by probes, as shown in Figure 7, we can see that $\mathrm{E}_{\mathrm{TJ}}=\mathrm{E}_{\mathrm{g}}$, confirming the absence of charging. Furthermore, in case of $\mathrm{h}=3 \mathrm{~mm}$, the flashover voltage was found to be 1.5 times higher [13].

In the above Figure 6, we have used a skirted cylindrical shape, but the charging control effect is also observed in the case when the spacer cuts into the end of the cathode. Figure 8 shows such a result, with the abscissa showing the height $\mathrm{h}$ of the insert region, and the ordinate showing the ratio of the charging component to the applied field $\left(\mathrm{E}_{\mathrm{s}} /\right.$ $E_{a v}$, with $E_{a v}$ as average electrical field; $E_{a v}=V_{a p} / d$. Note that the real geometrical field $\mathrm{E}_{\mathrm{g}}$ for this type of insulator is $\mathrm{k}$ times higher than $\mathrm{E}_{\mathrm{av}} . \mathrm{k}$ depends on the height $\mathrm{h}$, and also on the insert angle $\beta . \mathrm{k}$ is calculated to be $1.2-1.5$ in our

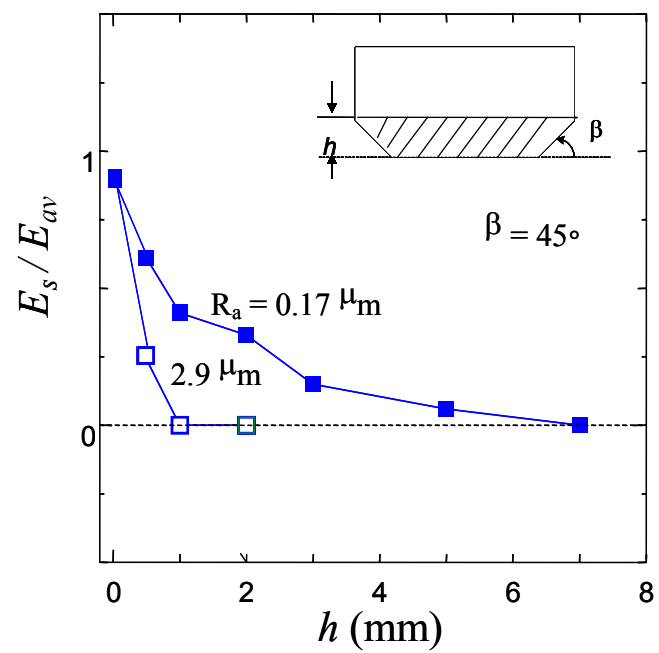

Figure 9. Relation of charge magnitude with $\mathrm{h}$ for roughened surface. (PMMA. $\beta=45^{\circ}, R_{a}$ of the surface of cylindrical part is $0.17 \mu \mathrm{m}$ ). 

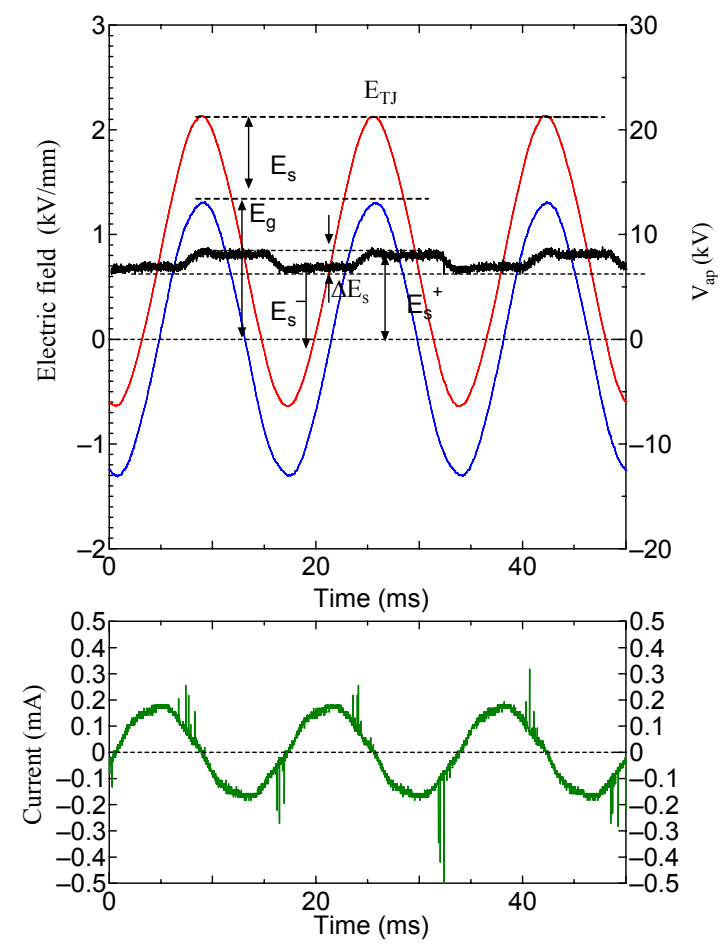

Figure 10. Charging phenomena of a cylindrical insulator under ac excitation. (PMMA. Cylindrical insulator, $\mathrm{R}_{\mathrm{a}}=0.17 \mu \mathrm{m} . \mathrm{h}_{1}=0$ ).

study.) As the graph shows, the ratio depends on $\beta$, but $E_{\mathrm{s}} /$ $E_{\text {av }}$ gets lower with the increase in $h$. For $\beta=45^{\circ}$, there is practically no charging effect for $\mathrm{h} \geq 5$.

When we combine the effect of spacer shape discussed above, and the effect of roughening the spacer surface, which was treated in Section 3, the results are shown in Figure 9. As seen from the graph, for the conical part having an average surface roughness of $R_{a}=2.9 \mu \mathrm{m}$, it is sufficient for it to have a height $\mathrm{h}=1 \mathrm{~mm}$, in order to achieve stable control of the charging effect [13].

In this experiment the distance between the charge and the probe increases with $\mathrm{h}$. However, we believe that the influence of increasing distance onto the charge measurements is limited, since it is deduced from the results with Figure 9 that the charging in this case takes place on the conical part of the surface.

\section{EXPERIMENTAL INVESTIGATION OF THE SHAPE EFFECT FOR AC VOLTAGE}

In this section, we consider the effect of the spacer shape in the case of ac voltage. For ac voltage, both the lower and the upper electrodes become cathode according to the phase, thus it is desirable to have conical sections on both ends of the cylindrical spacer. As an example, we consider the case of a spacer of $10 \mathrm{~mm}$ height, with both ends made conical, as shown later in the inserts of Figures 12 and 13, and the length $h_{1}$ of the conical sections is varied. The angle ( $\alpha$ or $\beta$ ) between the cathode surface and the spacer is kept constant at $\alpha=\beta=45^{\circ}$. In this experiment, the surface
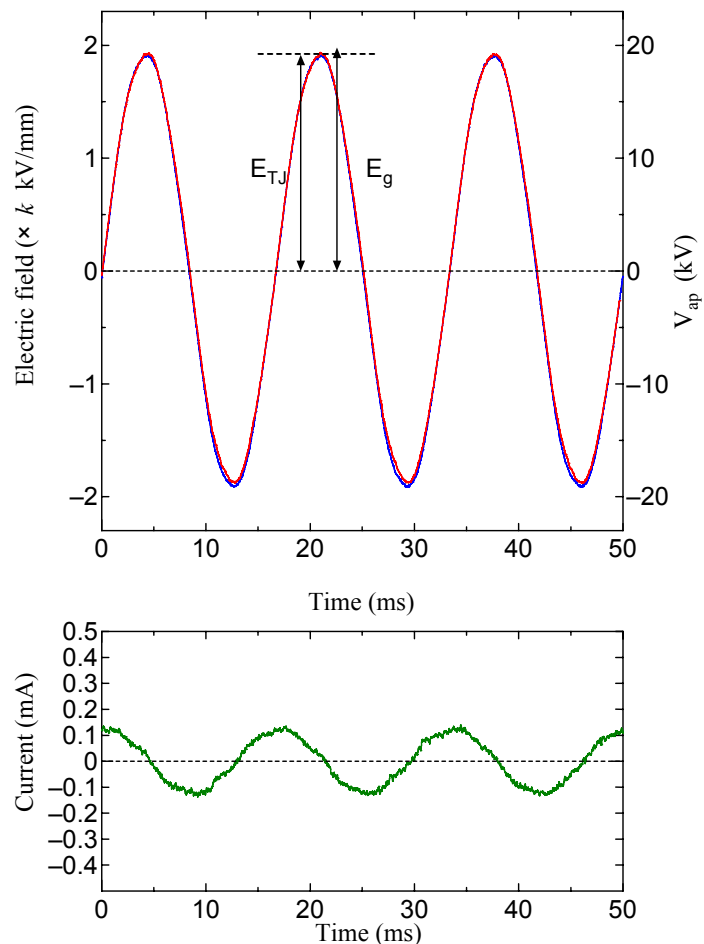

Figure 11. Probe signal showing that the charging is suppressed by adding conical sections at both ends. (ac. $\alpha=45^{\circ}, \mathrm{R}_{\mathrm{a}}=0.34 \mu \mathrm{m} . \mathrm{h}_{1}=3 \mathrm{~mm}$. The factor $\mathrm{k}$ of e-field axis is approximately 0.5 )

roughness of $\mathrm{R}_{\mathrm{a}}=0.34 \mu \mathrm{m}$ is employed. However, the change in charging effect due to the increase in surface roughness from $\mathrm{R}_{\mathrm{a}}=0.17 \mu \mathrm{m}$ to $0.34 \mu \mathrm{m}$ is not significant as it can be deduced from the results shown in Figure 4. Therefore, we believe the results with $0.34 \mu \mathrm{m}$ surface finish are practically the same when we analyze the effect of the conical sections.

First, the case of $\mathrm{h}_{1}=0$, that is, when a cylindrical spacer with no conical sections is directly exposed to ac voltage, is shown in Figure 10. The graph shows the charging component $\mathrm{E}_{\mathrm{s}}$, obtained by deducting the impressed field $\mathrm{E}_{\mathrm{g}}$ from the probe signal $\mathrm{E}_{\mathrm{TJ}}$. As seen from this $E_{s}$, the charging component has positive polarity, irrespective of the ac phase, that is, of the voltage polarity. The rising edge of the rectangular wave component occurs at the positive phase, while the falling edge at the negative phase and both are accompanied by current pulses. The charging process under ac excitation has already been discussed in our previous paper [10]. The charge depression effect due to surface roughening for ac voltage has been demonstrated also in the paper.

Experimental results for the case having conical sections on both ends of a cylindrical spacer are shown in Figure 11. In this graph, $\mathrm{E}_{\mathrm{TJ}}$ and $\mathrm{E}_{\mathrm{g}}$ overlaps completely. In other words, there is no charging effect. The same conclusion can be drawn from observing that there is no pulse component in the current, which shows only the ac component. 


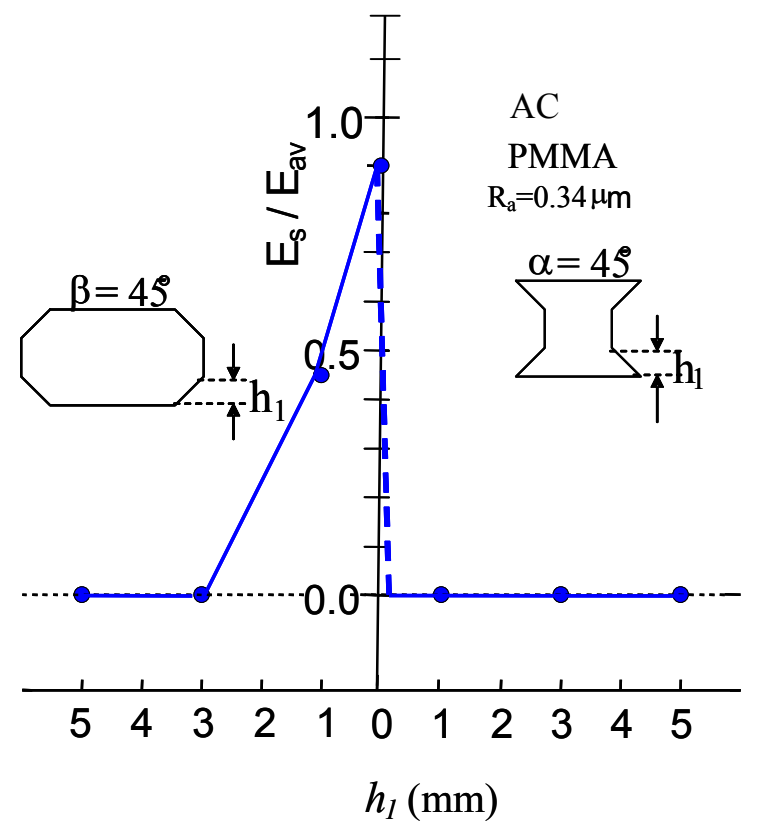

Figure 12. Charging characteristic of an insulator with conical base or cut at both ends.

Results showing the behavior of the charging, when the height of the conical part of spacers with conical shapes at both ends is varied, are shown in Figure 12. For conical base $\left(\alpha=45^{\circ}\right), \mathrm{h}_{1}=1 \mathrm{~mm}$ is sufficient to prevent the charging effect. For conical cut $\left(\beta=45^{\circ}\right)$, it is seen that $h_{1}$ $=3 \mathrm{~mm}$ is needed to suppress the charging effect. As seen from the results in Fiure.13, these methods succeed in raising the flashover voltage by a factor of more than 2 , by controlling the charging effect. The same graph shows that the conical cut shape has somewhat higher flashover voltage than the conical base.

\section{CONCLUSION}

We have investigated the effect of partial mechanical processing of an insulator on reducing the charging in vacuum. The truncated frustum or chamfered section of only a few mm high is found to be effective to suppress the charging under ac and dc voltage excitations. We have also found that the method of roughening a part of an insulator surface to a few microns, besides being a particularly simple way, is also effective in controlling the charging effect to the same extent, or even better, than the method of modifying the insulator shape.

Recently, the method of roughening an insulator surface was found to be effective in the wide range of insulating distances between a few hundreds of $\mu \mathrm{m}$ to $50 \mathrm{~mm}$ [14]. In the future, we intend to work on the practical application of these research results to the development of more compact and reliable equipment.

\section{REFERENCES}

[1] Application and Control of Discharge in Vacuum, Technical Report of the Japan Institute of Electrical Engineers, No. 586, 1996 (in Japanese).

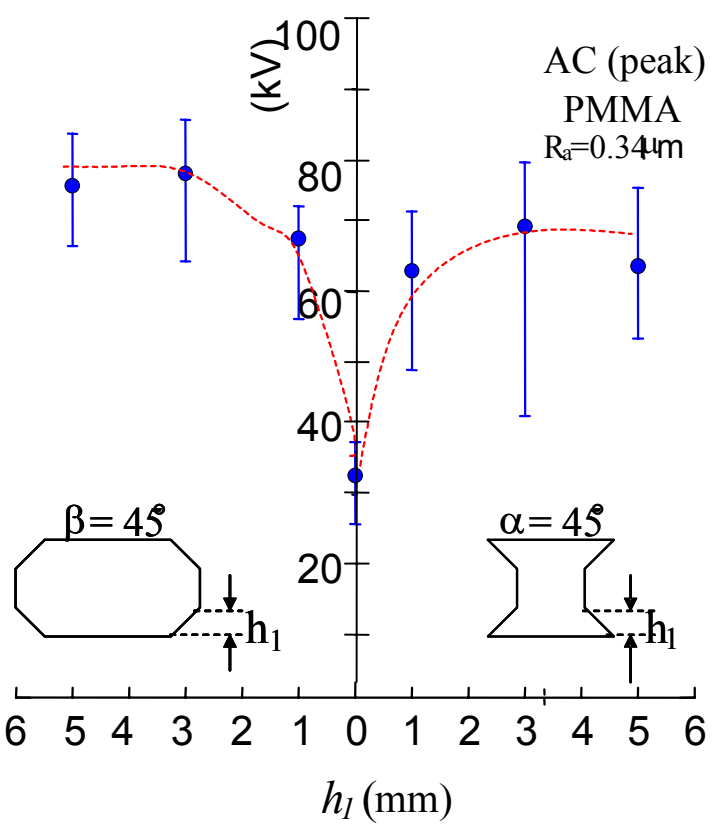

Figure 13. Flashover characteristic of machined insulator for ac voltage.

[2] Control Technology of Vacuum Discharge under High Energy Density Conditions, Technical Report of the Japan Institute of Electrical Engineers, No. 1001, 2005 (in Japanese).

[3] C. H. De Tourreil and K. D. Srivastava, "Mechanism of Surface Charging of High-Voltage Insulators in Vacuum", IEEE Trans. Electr. Insul, Vol. 8, pp.17-21, 1973.

[4] T. S. Sudarshan and J. D. Cross, "The Effect of Chromium Oxide Coatings on Surface Flashover of Alumina Spacers in Vacuum", IEEE Trans. Electr. Insul., Vol. 11, pp.32-35, 1976.

[5] A. Watson, "Pulsed Flashover in Vacuum", J. Appl. Phys., Vol. 38, pp.2019-2023, 1967.

[6] H. C. Miller, "Flashover of Insulators in Vacuum", IEEE Trans. Electr. Insul. Vol. 28, pp. 512- $527,1993$.

[7] O. Yamamoto, T. Takuma and Y. Tanabe, "Real-time observation of surface charging on a cylindrical insulator in vacuum", IEEE Trans. Dielectr. Electr. Insul., Vol.5, pp. 961-965, 1998.

[8] I. D. Chalmers, J. H. Lei, B. Yang and W. H. Siew, "Surface Charging and Flashover on Insulators in Vacuum", IEEE Trans. Dielelectr. Electr. Insul.,Vol, 2, pp.225-, 1995.

[9] O. Yamamoto, T. Takuma, M. Fukuda and S. Nagata, "Controlling Surface Discharge Path in Vacuum by Mechanical Processing on the Insulator Surface", IEEJ Trans. FM, Vol.123, NO.4, pp.370-375, 2003 (In Japanese).

[10] O. Yamamoto, S. Hamada, T. Fukuda, H. Omura, "Charging Characteristics of a Solid Insulator in Vacuum under ac Voltage Excitation", IEEE Trans. on Diele. Electr. Insul., Vol. 13, pp. 2-9, 2006.

[11] O. Yamamoto, T. Takuma, M. Fukuda, S. Nagata and T. Sonoda, "Improving Withstand Voltage by Roughening the Surface of an Insulating Spacer Used in Vacuum", IEEE Trans. Dielelectr. Electr. Insul., Vol. 10, pp. 550-556, 2003.

[12] O. Yamamoto, T. Takuma, Y. Kakehashi and S. Ikoma, "Charging Characteristics of a Conical Insulating Spacer in VacuumComparison of measured and theoretical results -, IEEJ, Vol. 120-A, No. 12, pp. 1070-1075, 2000 (In Japanese).

[13] O. Yamamoto, M. Fukuda, T. Takuma and T. Sonoda, "Influence of Space Shape on Charging and Flashover Characteristics in Vacuum", Trans. IEEDJ, Vol. 45, No.3 , pp.45-50, 2002 (In Japanese).

[14] O. Yamamoto, T. Satoh, H. Hayashi, S. Hamada, T. Kobayashi, H. Omura and H. Morii, "Flashover Characteristics of a Long Solid Insulator in Vacuum", Proc. of the XIVth Intel. Symposium on High Voltage Engineering, Paper NO. C-06, 2005 


\section{O. Yamamoto et al.: Depression of Insulator Charging in Vacuum by Partial Mechanical Processing}

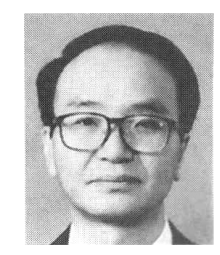

Osamu Yamamoto (M'02) was born on 22 February 1948 in Japan. He received the B.S. degree in physics from Ritsumeikan University in 1970 and the Dr. Degree in electrical engineering from Kyoto University in 1982 . He joined Kyoto University in 1971 as a research associate. He has been engaged in studies of High Voltage engineering especially on insulation characteristics and discharge mechanisms in gases including pressurized

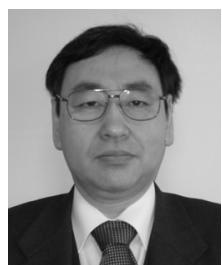

Hiroshi Morii was born in 1957. He graduated from Doshisha University, Kyoto Prefecture and joined The Kansai Electric Power Co. Inc. (KEPCO), Osaka, Japan in 1981. He has been engaged in Power Engineering R\&D Center of KEPCO since July, 2003. gas mixtures, surface flashover characteristics and mechanisms in vacuum. $\mathrm{He}$ is also interested in the gas separation and recovery technique from insulation gas mixtures and also investigations on the surge response of power transmission tower.

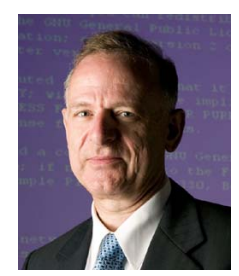

Sandor Markon (M'94) received the Dipl.Ing. degree in electrical engineering from Budapest Technical University in 1973. Joined Ganz Electric Works as a researcher, working on electromagnetic field analysis in large transformers and turbine generators. Postgraduate research student at Kyoto University from 1975 to 1977. Joined Fujitec Co., Ltd. of Japan in 1979, and had been working in applied research of computational intelligence, systems analysis and control. Received the Ph.D. degree from Kyoto University in 1996. Professor at Kobe Institute of Computing since 2005. Dr. Sandor Markon is a member of INNS and JNNS. 